\title{
Gpr120 agonist has anti-inflammatory and insulin-sensitizing effects
}

A team of researchers from the USA has developed a novel small-molecule Gpr120 agonist, cpdA, that has anti-inflammatory and insulin-sensitizing effects similar to $\omega-3$ fatty acids in obese mice.

$\omega-3$ fatty acids are known to have antiinflammatory effects and to be able to increase insulin sensitivity. In humans, GPR120 is the functional receptor for these fatty acids. "As the amount of fish oils (enriched $\omega-3$ fatty acids) that would have to be consumed to sustain chronic agonism of GPR120 is too high to be practical, a high affinity, small molecule GPR120 agonist would be of potential clinical

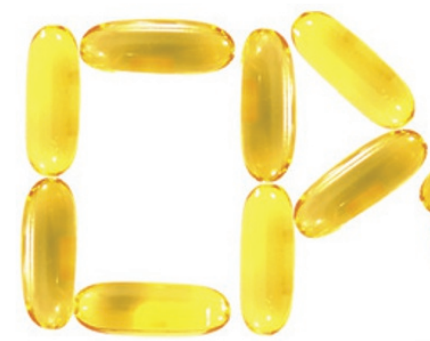

Polaris6D/iStock/Thinkstock/Modified by NPG benefit," explains author Da Young Oh (University of California San Diego, USA). None of the agonists investigated so far have been effective, which led $\mathrm{Oh}$ and colleagues to develop cpdA.

The researchers treated macrophages from wild-type and Gpr120-knockout mice with cpdA or docosahexaenoic acid (an $\omega$-3 fatty acid) for $1 \mathrm{~h}$, followed by stimulation with lipopolysaccharide. The two treatments had similar affects, inhibiting the phosphorylation of inflammatory kinases that is usually induced by lipopolysaccharide stimulation. Cytokine secretion and inflammatory gene expression were also inhibited in the wildtype mice but not in the Gpr120knockout mice. The metabolic effects in vivo were then determined using wild-type and Gpr120knockout mice on a high-fat diet alone or with cpdA added to the high-fat diet. The wild-type mice that received cpdA had improved glucose tolerance and insulin tolerance and decreased insulin secretion compared with mice on the high-fat diet alone; no difference was seen in the Gpr120-knockout mice on the two regimens.

These findings suggest that cpdA is a selective and potent activator of Gpr120, and that its effects are similar to those of $\omega-3$ fatty acids. "These GPR120 agonists could become future insulin sensitizing agents for the treatment of type 2 diabetes mellitus and other human insulin resistant states," concludes Oh.

\section{Claire Greenhill}

Original article Oh, D. Y. et al. A Gpr120-selective agonist improves insulin resistance and chronic inflammation in obese mice. Nat. Med. doi:10.1038/nm.3614 